\title{
SERIA A MACROFAUNA BENTÔNICA DE FUNDOS NÃO CONSOLIDADOS INFLUENCIADA PELO AUMENTO NA COMPLEXIDADE ESTRUTURAL DO HABITAT? O CASO DO ESTUÁRIO DA LAGOA DOS PATOS
}

\author{
ROSA, L.C. * \& BEMVENUTI, C.E. \\ Departamento de Oceanografia, Fundação Universidade Federal do Rio Grande (FURG), Caixa \\ Postal 474, Rio Grande, RS, Brasil, CEP 96201-900. \\ *Autor para correspondência (cielcr@furg.br)
}

\begin{abstract}
Rosa, L.C. \& Bemvenuti, C.E. 2007. Would be the benthic macrofauna of the soft-bottoms influenced by increase in the habitat structural complexity? The Patos Lagoon estuary as a case study. Braz. J. Aquat. Sci. Technol. 11(1):51-56. ISSN 1808-7035. The main objective of this study was to evaluate the influence of vegetated beds on macrofauna community structure of the estuarine soft bottoms of the Patos Lagoon, southern Brazil. Comparative analyses were made by faunal samples collected inside and outside in two kinds of vegetated beds: a sea grass meadows during the summer period and, an algal mats at winter one. The presence of theses vegetated beds had essentially favoring highest densities of epifaunal organisms by increase of substratum for fixation and feeding, which could to result at decrease of evenness and, consequently, of the diversity of macrofauna inside these beds such as registered at summer survey. Differences in the both evenness and diversity at winter survey as well in the composition and number of species at both times were not observed among habitats. These results indicate that the increase at structural complexity of habitat promoted by presence of vegetated beds not necessarily favoring the increase of the macrofauna diversity of the estuarine region of the Patos Lagoon.
\end{abstract}

Key-words: macrofauna structure, seagrass meadows, algal mats, habitat complexity, soft-bottoms, Patos Lagoon estuary

\section{INTRODUÇÃO}

Em ambientes de fundos não consolidados, a presença de vegetação resulta num aumento da complexidade estrutural do habitat, favorecendo com isso, um aumento na diversidade e na abundância da fauna aquática. Isso ocorre, essencialmente, devido a uma combinação de fatores tais como o aumento de área para fixação e para alimentação e a proteção contra predadores (Heck-Jr et al., 1995; Bostrom \& Bonsdorff, 1997).

No estuário da Lagoa dos Patos, extensos planos de águas rasas podem ser colonizados por fanerógamas aquáticas, sendo que Ruppia maritima L. é a espécie mais conspícua (Seeliger, 1998). A ocorrência, a persistência e o tamanho das pradarias de Ruppia, bem como sua biomassa e produção, variam espaçotemporalmente dependendo das condições ambientais locais (Seeliger, 1998). A maioria das pradarias de $R$. maritima possuem um ciclo anual, com elevada biomassa ocorrendo durante o verão (Costa \& Seeliger, 1989). Após a senescência da pradaria, esses fundos estuarinos podem ser colonizados por manchas de macro-algas, especialmente por clorófitas do gênero Ulva (Coutinho \& Seeliger, 1986).

$\mathrm{Na}$ Lagoa dos Patos, a influência da presença de fundos vegetados sobre a estrutura da comunidade estuarina ainda é um tema bastante negligenciado. Existem trabalhos publicados apenas sobre presença de pradarias de $R$. maritima, nos quais as assembléias de peixes (Garcia \& Vieira, 1997) e de crustáceos (Garcia et al., 1996; Kapusta \& Bemvenuti, 1998) dentro e fora desse ambiente foram comparadas. Embora já tenha sido o tema de alguns estudos (p.ex.: Asmus, 1984; Geraldi, 1997; Souza, 2001), dados publicados sobre a influência dessas pradarias bem como de outros tipos de fundos vegetados (i.e., fundos de macro-algas) sobre a estrutura do macrozoobentos estuarino (infauna e epifauna sedentária) ainda são inexistentes.

Desta forma, objetiva-se neste trabalho avaliar como um aumento na complexidade estrutural do habitat, promovido pela presença de fundos vegetados, influencia o macrozoobentos estuarino de fundos não consolidados da Lagoa dos Patos. Para tal, a estrutura da comunidade no interior de uma pradaria de Ruppia marítima (durante o verão) e de um fundo de macroalgas Ulva spp. (inverno) foi comparada as dos planos não vegetados adjacentes.

\section{MATERIAL E MÉTODOS}

O trabalho foi realizado numa enseada rasa (< $0,8 \mathrm{~m}$ de profundidade) localizado próxima à margem 
leste da Ilha das Pombas, região estuarina da Lagoa dos Patos ( $\left.32^{\circ} 01^{\prime} 505^{\prime \prime} \mathrm{S}, 052^{\circ} 07^{\prime} 708^{\prime \prime} \mathrm{W}\right)$. Neste local o sedimento é composto por areia muito fina com proporções de frações finas (i.e., silte e argila) variando entre 10 e $20 \%$, e o conteúdo de matéria orgânica, entre 0,5 e $2 \%$ (Rosa \& Bemvenuti, 2006).

Nessa região foram estabelecidas duas áreas de aproximadamente $10 \mathrm{~m}^{2}$ cada, sendo uma dentro e a outra fora dos fundos vegetados, as quais foram amostradas durante o verão (na presença de Ruppia maritima) e o inverno (na presença de Ulva sp.) de 2000. Em cada área, foram coletadas 12 amostras biológicas com o auxílio de um "corer" de PVC $\left(0,008 \mathrm{~m}^{2}\right.$ de área e $20 \mathrm{~cm}$ de profundidade), as quais foram peneiradas "in situ" através de uma malha de $0,3 \mathrm{~mm}$ de abertura e, o material retido, fixado com formaldeído $4 \%$. Em laboratório, os organismos foram triados, identificados até o menor nível taxonômico possível, quantificados e conservados em álcool $70 \%$.

O tratamento estatístico baseou-se em análises uni- e multivariadas, realizadas separadamente para cada situação (verão e inverno). Os descritores da comunidade utilizados foram o número de espécies, a densidade total (ind. $/ 0,008 \mathrm{~m}^{2}$ ) e os índices de equitabilidade de Pielou (J') e o de diversidade de Shannon-Wiener ( $\left.H^{\prime}\right)$ com logaritmo na base (e). Diferenças nesses parâmetros biológicos entre os fundos vegetados e não vegetados foram testadas através de uma análise de variância unifatorial, sendo que os dados foram testados para homocedasticidade (Teste de Cochran) e normalidade da variância (Teste de Kolmogorov-Smirnov) a priori de sua utilização na análise (Zar, 1984). Valores de número de espécies e de densidade obtidos durante o verão foram transformados pela raiz quarta $(O ̈ O ̈(x))$ para assumir os pressupostos da análise (Zar, 1984).

Posteriormente, os dados biológicos, transformados pela raiz quadrada, foram ordenados através de MDS, utilizando o índice de similaridade de Bray-Curtis (Clarke \& Warwick, 1994), e diferenças significativas entre a estrutura da macrofauna entre os hábitats foram testadas "a priori" através de uma análise de similaridade (i.e., ANOSIN; Clarke, 1993). Nos casos em que a ANOSIM indicou diferenças significativas, a contribuição de cada espécie para a dissimilaridade média entre os ambientes foi determinada através da análise de SIMPER (Clarke \& Warwick, 1994).

\section{RESULTADOS}

Nas coletas realizadas durante o verão dentro e fora da pradaria de $R$. maritima foram coletados um total de 6.456 indivíduos representados por 12 espécies. O gastrópode Heleobia australis foi o organismo mais abundante correspondendo a mais de $56 \%$ do total de organismos coletados. Outras espécies menos abundantes foram os poliquetas Laeonereis acuta $(11,7 \%)$, Heteromastus similis $(8,9 \%)$ e Nephtys fluviatilis $(1,8 \%)$, os isópodes Uromunna peterseni $(9,1 \%)$ e Kupellonura sp. $(0,3 \%)$, o anfípoda Mellita mangrovi (2,4\%), o tanaidáceo Kalliapseudes schubartii (6,6\%), os bivalves Erodona mactroides (1,9\%) e Tagelus plebeius $(0,1 \%)$, o cumáceo Diastylis simpterigiae $(0.5 \%)$ e um nemertino não identificado $(0,1 \%)$.

O número de espécies dentro $(8,75 \pm 0,75)$ e fora $(8,17 \pm 1,11)$ da pradaria foram estatisticamente semelhantes $(F=2,23 ; p=0,15)$. No entanto, maiores densidades foram registradas dentro da pradaria $(436,25$ $\pm 249,94$ ind. $\left./ 0,008 \mathrm{~m}^{2}\right)$ do que no plano não vegetado $\left(101,75 \pm 38,17\right.$ ind. $\left./ 0,008 m^{2} ; F=39,48 ; p<0,001\right)$. Por outro lado, os valores de equitabilidade e de diversidade da macrofauna foram significativamente menores dentro da pradaria $\left(J^{\prime}=0,62 \pm 0,12\right.$ e $\left.H^{\prime}=1,33 \pm 0,25\right)$ do que no plano não vegetado $\left(J^{\prime}=0,77 \pm 0,07 ; F=14,8 ; p<\right.$ 0,001 e $H^{\prime}=1,61 \pm 0,25 ; F=10,33 ; p=0,004$, respectivamente).

O resultado da análise de similaridade também indicou diferenças significativas (ANOSIM: $R=0,718$; $p=0,001)$ na estrutura da macrofauna entre os dois ambientes, as quais podem ser visualizadas pela formação de dois grupos distintos no MDS (Fig. 1), onde as amostras coletadas dentro da pradaria ficaram agrupadas no lado esquerdo do diagrama, enquanto que, as amostras do fundo não vegetado agruparam-se à direita do diagrama.

Juntas, elevadas densidades de $H$. australis, $U$. peterseni, $M$. mangrovii e $L$. acuta dentro da pradaria (Fig. 2) foram responsáveis por mais de $66 \%$ da dissimilaridade total entre os dois ambientes (Tabela 1).

Na amostragem realizada durante o inverno foram coletadas um total de 3.789 indivíduos nas amostras

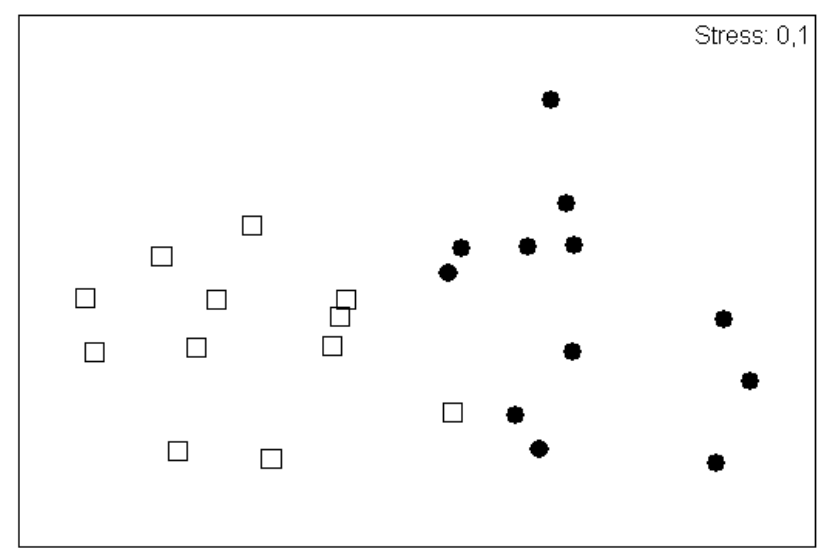

Figura 1 - MDS dos dados biológicos, transformados pela raiz quadrada, coletados dentro da pradaria de R. maritima (\%) e no fundo não vegetado adjacente (\%) durante o verão. 


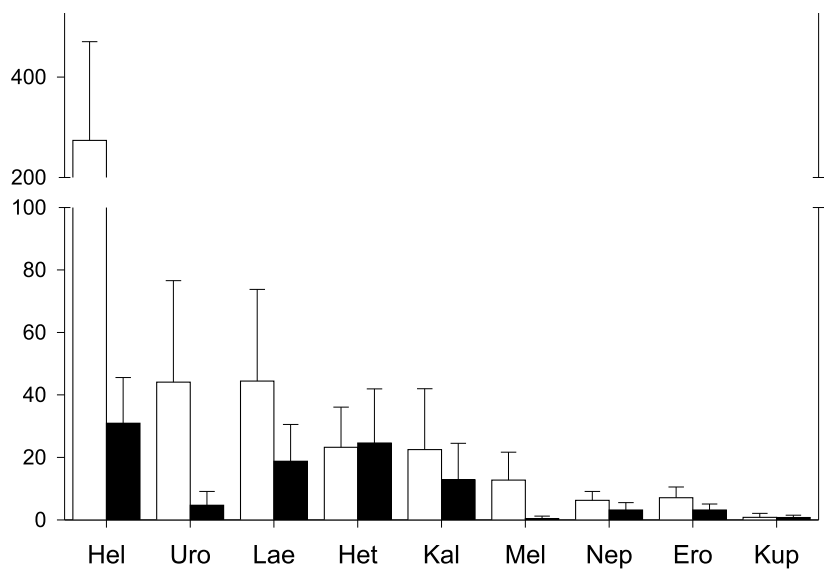

Figura 2 - Densidade média dos organismos macrofaunais coletados dentro da pradaria de $R$. maritima (colunas brancas) e no fundo não vegetado adjacente (colunas pretas) durante o verão. $\mathrm{Hel}=$ Heleobia australis, Uro = Uromunna peterseni, Lae = Laeonereis acuta, Het = Heteromastus similis, Kal = Kalliapseudes schubartii, $\mathrm{Mel}=$ Mellita mangrovi, Nep = Nephtys fluviatilis, Ero = Erodona mactroides, Kup = Kupellonura sp.

Tabela 1 - Contribuição individual das espécies para a dissimilaridade média $(\bar{\delta})$ entre os fundos não vegetados e os fundos de Ruppia maritima, ordenados por ordem de importância com um corte de $70 \%$.

\begin{tabular}{lcccc}
\hline \hline Espécies & $\overline{\boldsymbol{\delta}}$ & $\overline{\boldsymbol{\delta}} / \mathbf{D P}$ & Contribuição (\%) & Cumulativa (\%) \\
\hline H. australis & 13.46 & 2.18 & 32.37 & 32.37 \\
U. peterseni & 6.27 & 1.97 & 15.07 & 47.44 \\
M. mangrovii & 4.31 & 2.36 & 10.36 & 57.80 \\
L. acuta & 3.56 & 1.60 & 8.57 & 66.37 \\
\hline \hline
\end{tabular}

realizadas dentro e fora do banco de Ulva spp. e, novamente o gastrópode $H$. australis foi o organismo mais abundante correspondendo a $50 \%$ do total organismos. A composição da macrofauna também foi semelhante à observada no verão, exceto pela ausência do nemertino.

Semelhante ao registrado durante o verão, não foi observado diferença significativa no número de espécies entre os hábitats (dentro $=9,17 \pm 0,72$ e fora $=$ $8,67 \pm 0,78 ; F=2,67 ; p=0,116)$ e, maiores densidades foram registradas dentro do banco de Ulva $(197,92$ $\pm 41,51$ ind. $\left./ 0,008 \mathrm{~m}^{2}\right)$ do que no fundo não vegetado $\left(133,58 \pm 40,21\right.$ ind. $\left./ 0,008 m^{2} ; F=14,87 ; p<0,001\right)$. Por outro lado, os valores de equitabilidade e de diversidade da macrofauna dentro $\left(\mathrm{J}^{\prime}=0,66 \pm 0,10\right.$ e $\mathrm{H}^{\prime}=1,47 \pm$ $0,24)$ e fora do banco de macro-alga $\left(J^{\prime}=0,69 \pm 0,05 \mathrm{e}\right.$ $\left.H^{\prime}=1,41 \pm 0,13\right)$ não foram estatisticamente significantes $(F=0,96 ; p=0,337$ e $F=0,82 ; p=0,777$, respectivamente).

Diferenças na estrutura da macrofauna (ANOSIM: $R=0,491 ; p=0,001)$ entre os dois ambientes podem ser visualizadas na ordenação de MDS (Fig. 3). Novamente, maiores densidades de $H$. australis, $M$. mangrovii, $L$. acuta e Kupellonura sp. dentro do fundo de algas do que no fundo sem vegetação (Fig. 4) foram

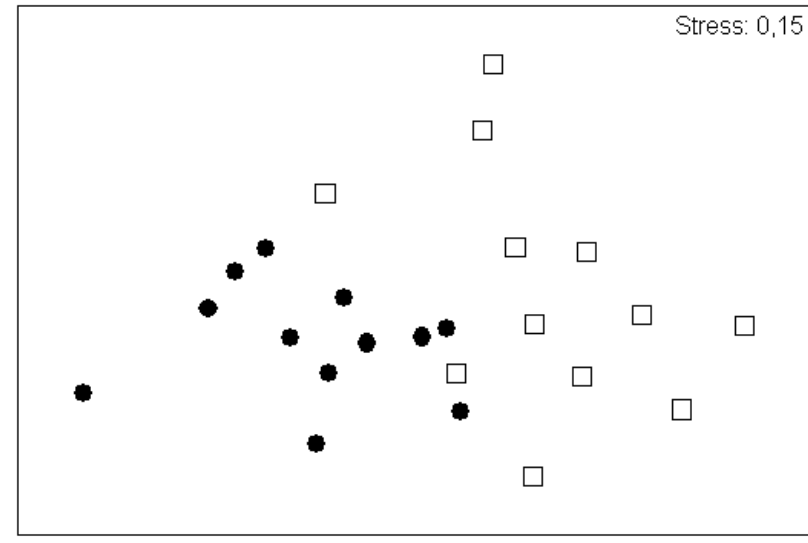

Figura 3 - MDS dos dados biológicos, transformados pela raiz quadrada, coletados dentro do banco de Ulva spp. (\%) e no fundo não vegetado adjacente (\%) durante o inverno.

responsáveis por mais de $56 \%$ da dissimilaridade total (Tabela 2).

\section{DISCUSSÃO}

Embora os dois tipos de vegetação sejam estruturalmente distintos (i.e., uma macro-alga e uma fanerógama) e tenham ocorrido em períodos distintos, verão e inverno, os exemplares da infauna e epifauna sedentária responderam de forma semelhante à presença da vegetação.

Exceto no verão, onde a equitabilidade e a diversidade da macrofauna foram menores no interior da pradaria de $R$. maritima, as semelhanças observadas na estrutura da comunidade entre os dois ambientes quanto a composição, número de espécies e os índices acima citados sugerem que a presença desses fundos vegetados não necessariamente resultará num aumento na diversidade da macrofauna estuarina da Lagoa dos Patos. Esses resultados coincidem com prévias observações obtidas sobre a influência da presença de pradarias de $R$. maritima sobre a macrofauna estuarina da região (Asmus, 1984; Geraldi, 1997; Rosa-Filho \& Bemvenuti, 1998).

Essa ausência de um incremento na diversidade em função de um aumento na complexidade do hábitat está provavelmente relacionada a dois fatores: a baixa diversidade com que a macrofauna na ocorre na região e, as características das pradarias de Ruppia marítima e dos bancos de macro-algas na região estuarina da Lagoa dos Patos. A Lagoa dos Patos, com seu longo e estreito canal, é considerada a maior laguna "estrangulada" do mundo (sensu Kjerfve, 1986). As características de uma laguna estrangulada e a baixa amplitude de marés astronômicas (cerca de 0,5 m), fazem com que o regime de salinidade seja fortemente 


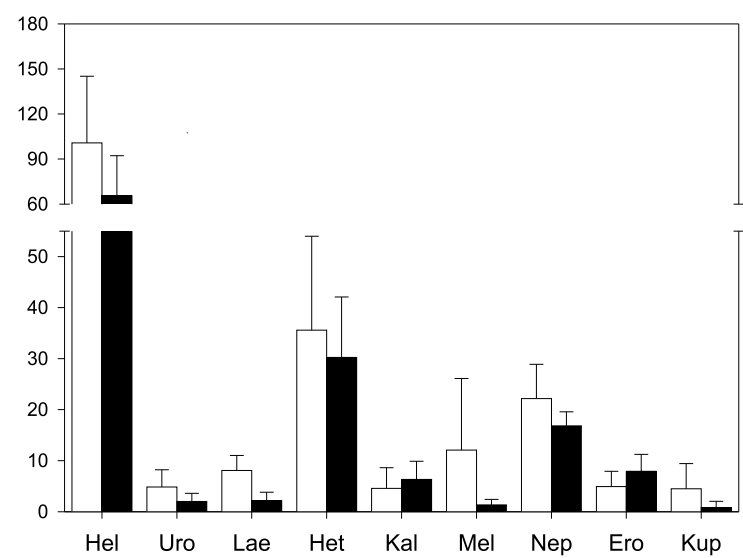

Figura 4 - Densidade média dos organismos macrofaunais coletados dentro do fundo de Ulva spp. (colunas brancas) e no fundo não vegetado adjacente (colunas pretas) durante o inverno. Hel = Heleobia australis, Uro = Uromunna peterseni, Lae = Laeonereis acuta, Het = Heteromastus similis, Kal = Kalliapseudes schubartii, Mel $=$ Mellita mangrovi, Nep $=$ Nephtys fluviatilis, Ero $=$ Erodona mactroides, Kup = Kupellonura sp.

influenciado pela ação dos ventos e da pluviosidade (Costa et al., 1998). Dessa forma, a alta variabilidade e a baixa previsibilidade dos parâmetros ambientais não permitem a formação de gradientes estáveis de salinidade, resultando assim em uma baixa diversidade de organismos na região estuarina (Bemvenuti et al., 1992). Essa alta instabilidade e baixa previsibilidade ambiental também afetam a ocorrência e a persistência desses fundos vegetados na região estuarina da laguna, dificultando ainda mais o desenvolvimento de uma comunidade específica para estes ambientes. Acompanhamentos de longo prazo que vem sendo realizados na região estuarina da Lagoa dos Patos (Projeto PELD - Pesquisas Ecológicas de Longa Duração - Site 8, Lago dos Patos), vem mostrando que a ocorrência dos fundos de $R$. maritima e de macroalgas na área em estudo caracteriza-se pela variabilidade interanual e baixa freqüência (obs. pessoal Carlos Bemvenuti).

Embora não tenha ocorrido um incremento na diversidade, a presença desses fundos favoreceu uma maior abundância da macrofauna, onde os valores de densidade foram de 1,5 a 4 vezes maiores no interior das áreas vegetadas por Ulva spp. e R. maritima, respectivamente, do que nos fundos não vegetados adjacentes.

Um maior incremento na abundância dos organismos no interior das pradarias de $R$. maritima está essencialmente relacionado a sazonalidade da macrofauna, do que propriamente às diferenças estruturais entre os dois tipos de fundos vegetados. Ainda mais que os fundos dominados por $R$. marítima não são mono-específicas, sendo comum no interior
Tabela 2 - Contribuição individual das espécies para a dissimilaridade média $(\bar{\delta})$ entre os fundos não vegetados e os fundos de Ulva spp, ordenados por ordem de importância com um corte de $70 \%$.

\begin{tabular}{lcccc}
\hline \hline Espécies & $\overline{\boldsymbol{\delta}}$ & $\overline{\mathbf{\delta}} / \mathbf{D P}$ & Contribuição (\%) & Cumulativa (\%) \\
\hline H. australis & 4.64 & 1.57 & 20.45 & 20.45 \\
M. mangrovii & 3.51 & 1.32 & 15.47 & 35.92 \\
L. acuta & 2.40 & 1.80 & 10.58 & 46.50 \\
Kupellonura sp. & 2.36 & 1.31 & 10.39 & 56.89 \\
\hline \hline
\end{tabular}

das pradarias a presença de massas de algas flutuantes tal como Ulva spp. (Seeliger 1998).

Na região, a macrofauna apresenta uma marcada variação sazonal, onde elevadas densidades observadas durante os meses de verão são resultante do processo reprodutivo dos organismos estimulado pelo aumento da temperatura a partir do final da primavera (Bemvenuti, 1997; Rosa \& Bemvenuti, 2006).

Durante esse período de recrutamento, uma maior disponibilidade de substrato para o assentamento e uma maior proteção contra os predadores proporcionados pela presença dessas pradarias, favoreceria o aumento da densidade da macrofauna no interior desses ambientes (Asmus, 1984; Bemvenuti, 1987).

Entre os organismos que se beneficiaram pela presença dos fundos vegetados e que tiveram uma maior contribuição para a dissimilaridade destes ambientes com os planos não vegetados está o gastrópode Heleobia australis. Este organismo epifaunal, que se alimenta do biofilme formado sobre o substrato, apresenta uma ampla distribuição batimétrica, ocorrendo desde os planos intermareais até as zonas profundas de canais com densidades superiores a 40.000 ind./ $\mathrm{m}^{2}$ (Bemvenuti, 1997). Na região, $H$. australis apresenta marcadas flutuações espaçotemporais, as quais estão relacionadas ao efeito dos predadores, à preferência por fundos arenolodosos e a facilidade de deslocamento da espécie (Bemvenuti et al., 1992; Bemvenuti, 1997). A relativa mobilidade e a capacidade de dispersão utilizando a tensão superficial da água permitem que $H$. australis rapidamente explore ambientes recém perturbados (Bemvenuti et al., 2003) bem como os fundos vegetados por pradarias ou macroalgas, os quais proporcionam, além de proteção, uma maior disponibilidade de hábitat e alimento para esse gastrópode (Bemvenuti, 1997).

Outros organismos que se beneficiaram pela presença dos fundos vegetados foram os crustáceos peracáridos como os isópodos Uromunna petersenie Kupellonura sp, e o anfípodo Mellita mangrovi. Estes organismos, todos integrantes da epifauna sedentária, os quais sofrem um forte controle populacional pela predação de juvenis de peixes e crustáceos decápodos, encontram nos fundos vegetados um refúgio contra a ação destes predadores (Bemvenuti, 1987). 
Desta forma, sendo a composição e o número de espécies iguais, um aumento nas densidades desses organismos oportunistas resultaria numa redução da equitabilidade e, conseqüentemente, da diversidade da macrofauna no interior dos fundos vegetados como observado na pradaria de $R$. maritima durante o verão.

Embora os resultados desse estudo confirmem prévias observações de que um aumento na complexidade estrutural devido a presença de fundos vegetados não resulta numa maior diversidade da macrofauna estuarina, mas favorece uma maior abundância de organismos epifaunais em seu interior, replicações espaço-temporais são necessários para melhor compreender a influência desses ambientes sobre os processos ecológicos locais. Diferenças estruturais e o efeito do tamanho desses fundos são também outros fatores que deverão ser considerados em estudos futuros.

\section{REFERÊNCIAS}

Asmus, M.L. 1984. Estrutura da comunidade associada a Ruppia maritima no estuário da Lagoa dos Patos, RS, Brasil. Tese de Mestrado. Fundação Universidade Federal do Rio Grande - FURG. 154p.

Bemvenuti, C.E. 1987. Predation effects on a benthic community in estuarine soft sediments. Atlântica, 9:5-32.

Bemvenuti, C.E. 1997. Benthic invertebrates. In: Seeliger, U.; Odebrecht, C. \& Castello, J.P. (ed.) Subtropical convergence environments: the coast and sea in the southwestern Atlantic. SpringerVerlag. 43-46pp.

Bemvenuti, C.E.; Cattaneo, S.A. \& Netto, S.A. 1992. Características estruturais da macrofauna bentônica em dois pontos da região estuarial da Lagoa dos Patos, RS, Brasil. Atlântica, 14:5-28.

Bemvenuti, C.E.; Rosa-Filho, J.S. \& Elliott, M. 2003. Changes in soft-bottom macrobenthic assemblages after a sulphuric acid spill in the Rio Grande Harbor (RS, Brazil). Brazilian Journal of Biology, 63:183194.

Bostrom, C. \& Bonsdorff, E. 1997. Community structure and spatial variation of benthic invertebrates associated with Zostera marina (L.) beds in the northern Baltic Sea. Journal of Sea Research, 37:153-166.

Clarke, K.R. 1993. Non-parametric multivariate analyses of changes in community structure. Australian Journal of Ecology, 18:117-143.

Clarke, K.R. \& Warwick, R.M. 1994. Changes in marine communities: an approach to statistical analyses and interpretation. Natural Environment Research Council, Plymouth, $144 \mathrm{p}$.

Costa, C.S.B. \& Seeliger, U. 1989. Vertical distribution and resource allocation of Ruppia maritima L. in a southern brazilian estuary. Aquatic Botany, 33:123129.

Costa, C.S.B.; Seeliger, U. \& Kinas, P.G. 1998. The effect of wind velocity and direction on the salinity regime in the lower Patos Lagoon estuary. Ciência e Cultura, 40:909-912.

Coutinho, R. \& Seeliger, U. 1986. Seasonal occurrence and growth of benthic algae in the Patos Lagoon Estuary, Brazil. Estuarine, Coastal and Shelf Science, 23:889-900.

Garcia, A.M. \& Vieira, J.P. 1997. Abundância e diversidade da assembléia de peixes dentro e fora de uma pradaria de Ruppia maritima L., no estuário da Lagoa dos Patos (RS, Brasil). Atlântica, 19:161181.

Garcia, A.M.; Vieira, J.P.; Bemvenuti, C.E. \& Geraldi, R.M. 1996. Abundância e diversidade da assembléia de crustáceos decápodos dentro e fora de uma pradaria de Ruppia maritima L. no estuário da Lagoa dos Patos (RS - Brasil). Nauplius, 4:113-128.

Geraldi, R.M. 1997. Características estruturais da assembléia de invertebrados bentônicos em fundos vegetados e não vegetados numa enseada estuarina da Lagoa dos Patos. Tese de Mestrado. Fundação Universidade Federal do Rio Grande - FURG. 208p.

Heck-Jr, K.L.; Able, K.W.; Roman, C.T. \& Fahay, M.P. 1995. Composition, abundance, biomass and production of macrofauna in a New England estuary: comparisons among eelgrass meadows and other nursery habitats. Estuaries, 18:379-389.

Kapusta, S.C. \& Bemvenuti, C.E. 1998. Atividade nictemeral de alimentação de juvenis de Callinectes sapidus, Rathbun, 1895 (Decapoda: Portunidae) numa pradaria de Ruppia maritima L. e num plano não vegetado, numa enseada estuarina da Lagoa dos Patos, RS, Brasil. Nauplius, 6:41-52.

Kjerfve, B. 1986. Comparative oceanography of coastal lagoons. In: Wolfe, D.A. (ed.) Estuarine variability. Academic Press, 63-81pp.

Rosa, L.C. \& Bemvenuti, C.E. 2006. Temporal variability of the estuarine macrofauna of the Patos Lagoon, Brazil. Revista de Biología Marina y Oceanografía, 41:1-9.

Rosa-Filho, J.S. \& Bemvenuti, C.E. 1998. Caracterización de las comunidades macrobentónicas de fondos blandos en regiones estuarinas de Rio Grande do Sul (Brasil). Thalassas, 14:43-56.

Seeliger, U. 1998. Fanerógamas marinhas submersas. In: Seeliger, U.; Odebrecht, C. \& Castello, J.P. (eds.) 
Rosa \& Bemvenuti: Macrofauna em fundos vegetados.

Os ecossistemas costeiro e marinho do extreme sul do Brasil. Ecoscientia. 29-32 pp.

Souza, R.A. 2001. Macroepifauna sedentária associada a macrófitas no Estuario da Lagoa dos Patos, RS,
Brasil. Tese de Mestrado, Fundação Universidade Federal do Rio Grande - FURG. 104 pp.

Zar, J.H. 1984. Biostatistical analysis. $2^{\text {nd }}$ Edition. Englewood Cliffs, Prentice Hall, New Jersey. 718 p. 